\title{
Predictors of pediatric readmissions among patients with neurological conditions
}

Ryan O'Connell ${ }^{1,2,3}$, William Feaster ${ }^{1,2}$, Vera Wang ${ }^{1,4}$, Sharief Taraman ${ }^{1,2,5}$ and Louis Ehwerhemuepha ${ }^{1,2,6^{*}}$ (D)

\begin{abstract}
Background: Unplanned readmission is one of many measures of the quality of care of pediatric patients with neurological conditions. In this multicenter study, we searched for novel risk factors of readmission of patients with neurological conditions.

Methods: We retrieved hospitalization data of patients less than 18 years with one or more neurological conditions. This resulted in a total of 105,834 encounters from 18 hospitals. We included data on patient demographics, prior healthcare resource utilization, neurological conditions, number of other conditions/diagnoses, number of medications, and number of surgical procedures performed. We developed a random intercept logistic regression model using stepwise minimization of Akaike Information Criteria for variable selection.

Results: The most important neurological conditions associated with unplanned pediatric readmissions include hydrocephalus, inflammatory diseases of the central nervous system, sleep disorders, disease of myoneural junction and muscle, other central nervous system disorder, other spinal cord conditions (such as vascular myelopathies, and cord compression), and nerve, nerve root and plexus disorders. Current and prior healthcare resource utilization variables, number of medications, other diagnoses, and certain inpatient surgical procedures were associated with changes in odds of readmission. The area under the receiver operator characteristic curve (AUROC) on the independent test set is $0.733(0.722,0.743)$.

Conclusions: Pediatric patients with certain neurological conditions are more likely to be readmitted than others. However, current and prior healthcare resource utilization remain some of the strongest indicators of readmission within this population as in the general pediatric population.
\end{abstract}

Keywords: Pediatric, Neurology, Readmission, Predictors, Indicators

\section{Background}

In pediatrics, the majority of health care costs can be attributed to a small group of patients. This group of patients is typically composed of children with medical complexities who require intensive care and are not easily catered to by existing models of health [1]. Improvements in the quality of care of these patients is

\footnotetext{
* Correspondence: lehwerhemuepha@choc.org

'Sharon Disney Lund Medical Intelligence and Innovation Institute (MI3) at CHOC, Orange, California, USA

${ }^{2}$ Children's Hospital of Orange County (CHOC), Orange, California, USA

Full list of author information is available at the end of the article
}

paramount to ensuring higher quality of life and reduced morbidity and mortality. Patients with neurological conditions largely contribute to this category as they often require complex medical care, making unplanned readmissions undesirable [2-4]. There is a dearth of research in risk factors and predictive models of unplanned readmission among these patients, so further investigation is a key step to improving quality of care and reducing corresponding readmission rates [5]. Reductions in unplanned readmissions may reduce financial distress, disruption in the families' and patients' lives, and help hospitals secure funding from organizations such as the Center for Medicare and Medicaid Services, which uses

(c) The Author(s). 2021 Open Access This article is licensed under a Creative Commons Attribution 4.0 International License, which permits use, sharing, adaptation, distribution and reproduction in any medium or format, as long as you give appropriate credit to the original author(s) and the source, provide a link to the Creative Commons licence, and indicate if changes were made. The images or other third party material in this article are included in the article's Creative Commons licence, unless indicated otherwise in a credit line to the material. If material is not included in the article's Creative Commons licence and your intended use is not permitted by statutory regulation or exceeds the permitted use, you will need to obtain permission directly from the copyright holder. To view a copy of this licence, visit http://creativecommons.org/licenses/by/4.0/ The Creative Commons Public Domain Dedication waiver (http://creativecommons.org/publicdomain/zero/1.0/) applies to the data made available in this article, unless otherwise stated in a credit line to the data. 
unplanned readmission rates as a proxy measure for hospital quality of care $[6,7]$.

Previous studies on unplanned readmission among patients with neurological conditions have indicated multiple anti-epilepsy drugs, pediatric intensive care unit admission, seizure with major complication or comorbidity, and presence of a major complication or comorbidity irrespective of diagnosis as factors associated with higher risk of unplanned readmission [3, 8]. In this study, we aimed to identify novel factors that may predict a higher risk of unplanned readmission in pediatric neurology and provide a prediction model that may be useful to clinicians in their efforts to reduce readmission rates $[9,10]$. Previous studies were limited by sample size and only included a single center. Using Cerner Health Facts Database, we were able to access data from more than 600 facilities across all care settings from participating Cerner Corporation clients in the United States. Results from this study may add to overall knowledge of unplanned readmissions in pediatric neurology, allowing for more targeted and higher quality of care.

\section{Methods}

We retrieved inpatient hospitalization data between 2000 and 2017 from the Cerner Health Facts Database of pediatric patients ( $<18$ years) with a nervous system condition as identified by diagnosis codes. The hospitals entered the study at different times and provided data over an average period of 9.2 years. We tagged each encounter as planned or unplanned using the diagnosis codes indicative of an encounter that is likely to be an aftercare or planned hospitalization. These codes included encounters for examination, encounters related to reproduction, encounters for specific health care needs such as chemotherapy, radiotherapy, surgery, and postprocedural aftercare, and encounters relating to certain mental health services, counselling, and care provider dependency corresponding to International Classification of Diseases Version 10 (ICD-10-CM) codes of Z00-Z13, Z30-Z39, Z40-Z53, and Z69-Z76 respectively. Earlier encounters used ICD-9-CM codes but were first converted to ICD-10-CM prior to our analyses of the data. We included only hospitals for which there was data in all of the database tables used for the study and for which there were at least 1000 encounters for a nervous system condition. We estimated the average age of all patients seen at each hospital and determined a freestanding pediatric hospital as one where the average age is less than 18 .

We estimated readmissions within 30 days and excluded encounters which preceded a planned readmission to the hospital within 30 days. This was to ensure that we were only comparing unplanned readmissions to encounters for which there was no readmission within
30 days. We chose the 30 -day metric over 7 days because it may provide increased opportunities to identify patients who potentially require interventions to improve quality of care and would help reduce the challenge in model performance due to class imbalance. We selected predictors encompassing the type/source of encounter, demographics, proxies for social determinants of health, sub-classes of neurological conditions, count of the number of other systems of diagnoses of each encounter, several measures of resource utilization (including histories of hospitalizations, readmissions, and emergency room/department visits), number of surgical procedures carried out during the visit, and the number of medications administered to the patient during the index visit. See Table 1 and the Additional file 1 for a complete list of variables considered during model development.

\section{Statistical considerations}

We excluded rare neurological conditions (toxic encephalopathy (G92), transient cerebral ischemic attacks and related syndromes (G45), vascular syndromes of brain in cerebrovascular diseases (G46), and other diseases of the nervous system without clear designation (G98-G99)) as well as rare surgical procedures (endocrine, eye/ocular adnexa, and mediastinum and diaphragm surgeries) as they present problems with model development due to statistical separability [11]. We assessed multicollinearity between predictors using the generalized variance inflation factor which estimates the degree to which variance estimates are inflated due to correlation between predictors [12].

We split the data into a training $(80 \%)$ and test set and developed mixed effects (random intercept) logistic regression model. We performed variable selection using a stepwise procedure to obtain the combination of predictors that result in the lowest value of the Akaike Information Criteria [13]. We assessed model performance by estimating the area under the operator characteristic curve, and values of sensitivity, positive predictive value, relative risk, and the number needed to evaluate at an a priori value of specificity at 0.90 . All analyses were carried out using HealtheDataLab and the R Statistical Programming Language [14, 15].

\section{Results}

The inclusion/exclusion criteria as discussed in the Methods resulted in 105,834 total index admissions from 18 hospitals that were evaluated in the analysis. This includes 12,737 30-day readmissions and corresponds to a $12.0 \% 30$-day readmission rate. The mean age of the patients was 8 years with a standard deviation of 6 years. There were 52.6\% males, 56.4\% Caucasians, $20.4 \%$ African American/Black, and 2.6\% patients of Hispanic ethnicity. The largest groups of patients were on Medicare/ 
Table 1 Demographics, resource utilization, and comorbid conditions

\begin{tabular}{|c|c|c|c|c|}
\hline Variables & Levels & $\begin{array}{l}\text { Not readmitted } \\
\text { n (\%) or mean (sd) }\end{array}$ & $\begin{array}{l}\text { Readmitted } \\
\text { n (\%) or mean (sd) }\end{array}$ & $\begin{array}{l}\text { chi- } \\
\text { squared } \\
\text { or t-test } \\
p \text { value }\end{array}$ \\
\hline Age & - & $6.75(6.09)$ & $7.08(5.97)$ & $<0.001$ \\
\hline \multirow[t]{2}{*}{ Sex } & Female & 71455 (46.02) & $8204(46.43)$ & 0.301 \\
\hline & Male & 83808 (53.98) & $9464(53.57)$ & \\
\hline \multirow[t]{6}{*}{ Race/ethnicity } & Caucasian & $88573(57.05)$ & $9582(54.23)$ & $<0.001$ \\
\hline & African American/ Black & 30498 (19.64) & $3415(19.33)$ & \\
\hline & Hispanic & $4185(2.70)$ & $586(3.32)$ & \\
\hline & Native American & $2119(1.36)$ & $199(1.13)$ & \\
\hline & Asian/ Pacific Islander & $2060(1.33)$ & $235(1.33)$ & \\
\hline & Other & $27828(17.92)$ & $3651(20.66)$ & \\
\hline \multirow[t]{4}{*}{ Payer } & Commercial & 47555 (30.63) & $5242(29.67)$ & $<0.001$ \\
\hline & Governmental & $67552(43.51)$ & $8836(50.01)$ & \\
\hline & Self pay & $2790(1.80)$ & $188(1.06)$ & \\
\hline & Others & $37366(24.07)$ & $3402(19.26)$ & \\
\hline \multirow[t]{4}{*}{ Length of stay (days) } & $<2$ & $37491(24.15)$ & $2708(15.33)$ & $<0.001$ \\
\hline & $2-3$ & $55240(35.58)$ & $5245(29.69)$ & \\
\hline & $4-6$ & $27874(17.95)$ & $3812(21.58)$ & \\
\hline & 7 or more & $34658(22.32)$ & $5903(33.41)$ & \\
\hline \multirow[t]{2}{*}{ Index visit is planned } & No & 123208 (79.35) & $14383(81.41)$ & $<0.001$ \\
\hline & Yes & $32055(20.65)$ & $3285(18.59)$ & \\
\hline \multirow[t]{2}{*}{ Admitted through ED } & No & $33912(21.84)$ & $4008(22.69)$ & 0.011 \\
\hline & Yes & $121351(78.16)$ & $13660(77.31)$ & \\
\hline \multirow[t]{3}{*}{ Index visit is a readmission? } & No & $139316(89.73)$ & $11881(67.25)$ & $<0.001$ \\
\hline & Yes, unplanned & $13042(8.40)$ & $4513(25.54)$ & \\
\hline & Yes, planned & $2905(1.87)$ & $1274(7.21)$ & \\
\hline \multirow[t]{4}{*}{ Previous ED visits (prior $6 \mathrm{mo}$ ) } & 0 & $118780(76.50)$ & $11369(64.35)$ & $<0.001$ \\
\hline & 1 & $22665(14.60)$ & $3239(18.33)$ & \\
\hline & 2 & $7696(4.96)$ & $1444(8.17)$ & \\
\hline & 3 or more & $6122(3.94)$ & $1616(9.15)$ & \\
\hline \multirow[t]{4}{*}{ Previous hospitalizations (prior 6mo) } & 0 & $119481(76.95)$ & $7959(45.05)$ & $<0.001$ \\
\hline & 1 & $22409(14.43)$ & $3673(20.79)$ & \\
\hline & 2 & $7097(4.57)$ & $2076(11.75)$ & \\
\hline & 3 or more & $6276(4.04)$ & $3960(22.41)$ & \\
\hline \multirow[t]{4}{*}{ Previous readmissions (prior $6 \mathrm{mo}$ ) } & 0 & $146664(94.46)$ & $13043(73.82)$ & $<0.001$ \\
\hline & 1 & $5441(3.50)$ & $2067(11.70)$ & \\
\hline & 2 & $1727(1.11)$ & $1076(6.09)$ & \\
\hline & 3 or more & $1431(0.92)$ & $1482(8.39)$ & \\
\hline Number of comorbid diagnoses (by ICD-10-CM chapters) & - & $5.35(5.22)$ & $6.73(6.12)$ & $<0.001$ \\
\hline
\end{tabular}

Medicaid health insurance (42.9\%) while $31.7 \%$ had Commercial insurance, with the remainder on other health insurance types or self-pay.

We assessed univariable (unadjusted) statistics and developed a corresponding multivariable model on the training dataset. All variables considered had univariable significance (unadjusted $p$ value $<0.05$ ) except sex, demyelinating diseases of the CNS (G35-G37), epilepsy (G40), intraoperative and postprocedural complications of the CNS (G97), migraine (G43), other degenerative diseases of 
the nervous system (G30-G32), other headache syndromes (G43), other and unspecified diseases of the spinal cord (G95), pain (G89), systemic atrophies primarily affecting the CNS (G10-G14), surgical procedures on the digestive system, integumentary procedures, and surgical procedures on the urinary and reproductive organs. We did not detect any severe problems with multicollinearity between predictors based on a rule of thumb threshold for excluding highly correlated variables [12].

Of neurological factors associated with a change in the odds of readmission within 30-days, the multivariate model indicates that the following are risk factors of readmission among patients with neurological conditions: malignant neoplasm of the brain (C71), polyneuropathies (G62), congenital hydrocephalus (G91), other amino acid metabolism disorders (E72), paralytic strabismus (H49), hydrocephalus (G91), other autosomal trisomies (Q92), neuromuscular dysfunction of bladder (N31), microcephaly (Q02), Down Syndrome (Q90), brain disorders (G93). On the other hand, there are reduced odds of readmission among patients with some neurological conditions (Table 2) including sleep disorders (G47), convulsions (R56), and intracranial injury (S06). The remaining neurological conditions identified did not have statistically significant effects on the risk/ odds of readmission in the multivariable model. The number of comorbid diagnoses (such as respiratory and circulatory systems of diagnoses) is associated with increased odds of readmission. In particular, patients undergoing chemotherapy during the index visit have highly elevated odds of readmission - these patients are being treated for some form of cancer as well as some neurological condition (that may or may not be related with the diagnosis for cancer).

Results from the multivariable model indicate that current and prior healthcare resource utilizations are most predictive of the risk of readmission after the index visit. Patients with longer lengths of stay are more likely to be readmitted than those with shorter lengths of stay, and the higher the number of previous hospitalizations (and the longer the lengths of stay of these previous hospitalizations) within the prior 6 months, the more likely a 30-day readmission will occur after the index/current hospitalization. Similarly, the risk of subsequent readmission is elevated if the index visit is a readmission within 30-days of the prior visit. The number of previous unplanned readmissions as well as emergency room/department visits within the prior 6 months are associated with increased risk of readmission, whereas the risk of readmission is reduced if the index visit is a planned encounter. These results are consistent with prior studies mostly in the general pediatric and adult populations $[3,10,16]$.

In this population of pediatric patients with neurological conditions, younger patients are more likely to be readmitted than their older peers. The type of admission is associated with a statistically significant change in the risk of readmission. Emergent admissions are associated with higher risk of readmission. In comparison to patients with Commercial insurance, self-pay patients are less likely to be readmitted.

The odds of readmission are increased among these patients with underlying neurological conditions if they are also undergoing nervous or cardiovascular system surgical procedures. On the other hand, surgical procedures on the musculoskeletal system are associated with reduced odds of readmission. The odds ratios and corresponding 95\% Confidence Intervals are given in Table 2.

The area under the receiver operator characteristics curve obtained in the independent test set was 0.733 (0.722, 0.743). Based on previous studies, we recommend setting the predicted probability threshold for flagging a patient at high risk of readmission (and requiring interventions) at a value that guarantees a model specificity of 0.90 (or $90 \%$ ). At this value of the specificity of the model, the sensitivity, positive predictive value, relative risk, and number needed to evaluate are $0.373(0.354$, $0.392), 0.339$ (0.321, 0.356), 3.878 (3.617, 4.157), and 3 respectively. This implies that we expect to capture $37 \%$ of readmissions in such a way that there will be 2 false positives for every 1 true positive prediction. The corresponding predicted probability of readmission should be set at 0.200 (nearly twice the baseline readmission rate of these patients).

\section{Discussion}

In this study, we identified several neurological conditions that increase the risk of readmission among pediatric neurology patients. Among the neurologic risk factors we identified, it is likely that higher readmission rates in these populations in part represents the disease burden and complex nature of presentation in these patients, rather than any specific diagnostic or therapeutic shortcomings. Specifically, a preponderance of these risk factors are chronic, lifelong diagnoses, or carry long term morbidity implications (microcephaly, Down Syndrome, metabolic disorders, etc.). This thought remains true when juxtaposed to the neurological conditions we identified to have lower odds of readmission (meningitis, intracranial injury, sleep disorders, etc.). Reduced odds of readmission among these patients may attributable to the fact that they tend to be time limited and are more frequently encountered in diagnostic isolation or with fewer comorbid complications. While many of the seemingly protective conditions can be deadly, we controlled for this by excluding patients that died during an admission. This shows one of our study limitations. We had no way of measuring the objective severity of an illness such as meningitis. An assumption could be made that 
Table 2 Multivariable Results

\begin{tabular}{|c|c|c|c|}
\hline Variable & Levels & Odds ratio & $p$ values \\
\hline Index Visit is Planned & Yes & $0.898(0.847,0.952)$ & $<0.001$ \\
\hline \multirow[t]{4}{*}{ Length of Stay (days) } & $<2$ & Reference & - \\
\hline & $2-4$ & $1.228(1.167,1.293)$ & $<0.001$ \\
\hline & $4-6$ & $1.441(1.361,1.525)$ & $<0.001$ \\
\hline & 7 or more & $1.585(1.492,1.683)$ & $<0.001$ \\
\hline Age & - & $0.993(0.99,0.997)$ & $<0.001$ \\
\hline \multirow[t]{6}{*}{ Race } & Caucasian & Reference & - \\
\hline & African American/Black & $0.992(0.946,1.04)$ & 0.729 \\
\hline & Hispanic/Latino & $1.126(1.016,1.247)$ & 0.024 \\
\hline & Native American & $0.855(0.696,1.05)$ & 0.136 \\
\hline & Asian/Pacific Islander & $1.1(0.948,1.276)$ & 0.208 \\
\hline & Other & $0.978(0.928,1.031)$ & 0.417 \\
\hline \multirow[t]{2}{*}{ Sex } & Female & Reference & - \\
\hline & Male & $1.017(0.983,1.052)$ & 0.336 \\
\hline \multirow[t]{4}{*}{ Payer } & Commercial & Reference & \\
\hline & Governmental & $1.028(0.986,1.073)$ & 0.196 \\
\hline & Self-Pay & $0.805(0.688,0.943)$ & 0.007 \\
\hline & Others & $1.031(0.972,1.092)$ & 0.309 \\
\hline \multirow[t]{4}{*}{ Previous ED Visits (prior 6 mo) } & 0 & Reference & - \\
\hline & 1 & $1.106(1.056,1.159)$ & $<0.001$ \\
\hline & 2 & $1.200(1.123,1.283)$ & $<0.001$ \\
\hline & 3 or more & $1.297(1.209,1.391)$ & $<0.001$ \\
\hline \multirow[t]{3}{*}{ Index Visit is a Readmission? } & No & Reference & - \\
\hline & Yes, Planned & $1.338(1.269,1.411)$ & $<0.001$ \\
\hline & Yes, Unplanned & $1.666(1.516,1.832)$ & $<0.001$ \\
\hline \multirow[t]{4}{*}{ Previous Hospitalizations (prior 6 mo) } & 0 & Reference & - \\
\hline & 1 & $1.695(1.613,1.781)$ & $<0.001$ \\
\hline & 2 & $2.348(2.18,2.528)$ & $<0.001$ \\
\hline & 3 or more & $3.014(2.738,3.317)$ & $<0.001$ \\
\hline \multirow[t]{4}{*}{ Previous Readmissions (prior 6 mo) } & 0 & Reference & - \\
\hline & 1 & $1.179(1.09,1.274)$ & $<0.001$ \\
\hline & 2 & $1.496(1.338,1.673)$ & $<0.001$ \\
\hline & 3 or more & $2.051(1.831,2.298)$ & $<0.001$ \\
\hline \multirow[t]{11}{*}{ Neurologic Risk Factors } & Malignant neoplasm of brain (C71) & $1.953(1.788,2.133)$ & $<0.001$ \\
\hline & Polyneuropathies (G62) & $1.5(1.248,1.803)$ & $<0.001$ \\
\hline & Congential Hydrocephalus (Q03) & $1.318(1.175,1.478)$ & $<0.001$ \\
\hline & Other AA Metabolism Disorders (E72) & $1.313(1.095,1.575)$ & 0.003 \\
\hline & Paralytic Strabismus (H49) & $1.288(1.124,1.476)$ & $<0.001$ \\
\hline & Hydrocephalus (G91) & $1.218(1.131,1.311)$ & $<0.001$ \\
\hline & Other Autosomal Trisomies (Q92) & $1.214(1.019,1.448)$ & 0.030 \\
\hline & Neuromuscular Dysfunction of Bladder (N31) & $1.155(1.034,1.29)$ & 0.011 \\
\hline & Microcephaly (Q02) & $1.131(1.005,1.272)$ & 0.041 \\
\hline & Down Syndrome (Q90) & $1.129(1.036,1.23)$ & 0.005 \\
\hline & Brain Disorders (G93) & $1.078(1.019,1.14)$ & 0.009 \\
\hline
\end{tabular}


Table 2 Multivariable Results (Continued)

\begin{tabular}{|c|c|c|c|}
\hline Variable & Levels & Odds ratio & $p$ values \\
\hline \multirow[t]{14}{*}{ Other Neurological Conditions } & Convulsions (R56) & $0.922(0.874,0.972)$ & 0.003 \\
\hline & Nervous \& Msuculoskeletal System Symptoms (R29) & $0.882(0.781,0.995)$ & 0.041 \\
\hline & Coginitive Function Symptoms (R41) & $0.853(0.776,0.938)$ & $<0.001$ \\
\hline & Sleep Disorders (G47) & $0.84(0.791,0.892)$ & $<0.001$ \\
\hline & Meningitis, Other Causes (G03) & $0.829(0.698,0.985)$ & 0.033 \\
\hline & Nystagmus \& Irregular Eye Movements (H55) & $0.825(0.686,0.992)$ & 0.040 \\
\hline & Newborn Cerebral Distrubances (P91) & $0.822(0.705,0.958)$ & 0.012 \\
\hline & Speech \& Language Development Disorders (F80) & $0.811(0.725,0.907)$ & $<0.001$ \\
\hline & Somnolence, Stupor, Coma (R40) & $0.786(0.694,0.889)$ & $<0.001$ \\
\hline & Mental Disorders Due to Physiological Condition (F06) & $0.768(0.648,0.91)$ & 0.002 \\
\hline & Bacterial Meningitis (G00) & $0.622(0.487,0.796)$ & $<0.001$ \\
\hline & Newborn Muscle Tone Disorders (P94) & $0.621(0.567,0.68)$ & $<0.001$ \\
\hline & Intracranial Injury (S06) & $0.476(0.418,0.543)$ & $<0.001$ \\
\hline & Viral Meningitis (A87) & $0.446(0.353,0.563)$ & $<0.001$ \\
\hline Number of Medications & - & $1.014(1.012,1.016)$ & $<0.001$ \\
\hline Free-standing pediatric hospital & Yes & $1.586(1.103,2.28)$ & 0.013 \\
\hline \multirow[t]{3}{*}{ Surgical procedures } & Cardiovascular & $1.204(1.109,1.307)$ & $<0.001$ \\
\hline & Musculoskeletal & $0.685(0.6,0.781)$ & $<0.001$ \\
\hline & Nervous System & $1.119(1.029,1.216)$ & 0.009 \\
\hline $\begin{array}{l}\text { Number of Comorbid Diagnoses } \\
\text { (by ICD-10-CM chapters) }\end{array}$ & - & $1.01(1.006,1.014)$ & $<0.001$ \\
\hline Emergent Admission & Yes & $1.129(1.079,1.18)$ & $<0.001$ \\
\hline
\end{tabular}

very severe presentations are less likely to leave the hospital until providers are exceedingly comfortable with the patient's recovery. Similarly, more complex presentations may lead to a lower likelihood of discharging a patient prematurely, accounting for the general trends in both the protective and risk related diagnoses we identified.

Other predictors of higher risk of unplanned readmission (among patients with underlying neurological conditions) in our multivariable model include longer length of stay, higher number of previous hospitalizations, and unplanned index visit. As representative markers of health care utilization these are consistent with findings from prior studies. Certain surgical procedures also predicted higher risk of unplanned readmission and included nervous and cardiovascular system procedures during hospitalizations. We also observed a reduced risk in patients with older age, self-pay coverage, and patients undergoing surgical procedures of the musculoskeletal system. The reduced risk from musculoskeletal procedures likely represents the specific limited nature of these encounters. Overall, current and prior healthcare resource utilizations are most predictive of risk of unplanned readmission after the index visit.
Previous studies have identified risk factors such as higher severity of illness, public health insurance, multiple antiepileptic drugs, intensive care admission, and seizures with major complication/comorbidity [3, 8]. These findings are generally consistent with the results of this study. Several proxies for severity of illness and comorbidity were all associated with increased risk of readmission. While one of the studies found that multiple antiepileptic drugs are associated with increased risk of readmission [3], our findings indicated that the higher the overall number of medications administered the higher the risk of readmission.

The aim of such research is to develop a model that may be helpful in improving our understanding of risk factors of readmission in pediatric neurology, and for predicting future risk of readmission in a clinical setting. We provided information on how this model may be implemented to assist decision making and allow for earlier interventions. Reducing unplanned readmission rates would benefit all parties involved by saving time and money and helping the patient avoid unexpected time in the hospital. Those who are determined to be at high risk according to this model may benefit from personalized interventions before and after discharge.

While the primary goal of implementing a predictive model such as this is to prevent readmission by 
providing additional information during an ongoing hospital stay, the results are also likely to be informative to ambulatory care providers post-discharge. A patient being flagged as high risk for readmission could help guide decision making at early post-discharge follow-up appointments, provided they are sharing information systems or interfaces with the admitting facility. Furthermore, the results should generalize to non-US healthcare settings with electronic medical records as the risk factors are not peculiar to US population.

A limitation of this study is that we could not track patients across hospitals. In other words, patients readmitted to a hospital different from the index encounter were likely not captured. This study limitation may also account for the finding that free standing pediatric hospitals are correlated with an increased risk of readmission. There are likely cases in which a pediatric patient was initially admitted at a general/adult facility are subsequently transferred to, and followed by, a dedicated pediatric facility for a higher level of care. This would be associated with a concentration of complex cases being encountered at dedicated pediatric facilities compared to non-pediatric facilities.

An additional minor limitation is the fact that the data consisted of health care systems using the Cerner EMR and who consented to sharing deidentified data. There is concern that an inability to reliably track death subsequent to discharge in our data set may impart some protective bias for some highly lethal disease states, however we have not clearly identified a suggestion of this bias in the model.

The large size of the study and distribution across the United States indicate that the findings may be helpful to a large number of pediatric neurology patients across the country. Furthermore, the opportunity of capturing $37 \%$ of unplanned readmissions with only 2 false positive for every true positive prediction is quite impressive. In other words, a large number of unplanned readmissions may be captured with high accuracy, allowing for focused interventions specifically on patients most in need of such interventions. The model specificity may be traded for higher sensitivity at hospitals where there are sufficient clinical resources for interventions. While a high false positive rate is present in our model, we focused specifically on readmissions, and further research is necessary to find if these false positive cases may be predictive of some other state that warrants additional consideration or intervention.

\section{Conclusions}

Pediatric patients with certain neurological conditions are more likely to be readmitted than others. However, current and prior healthcare resource utilization remain some of the strongest indicators of readmission within this population as in the general pediatric population. After accounting for prior healthcare utilization, pediatric patients with malignant neoplasm of the brain, congenital hydrocephalus, microcephaly, down syndrome and other brain disorders among others have a higher risk of unplanned readmission. Our findings will allow clinicians to identify patients most vulnerable to future unplanned readmissions and adjust their care accordingly.

\section{Supplementary Information}

The online version contains supplementary material available at https://doi. org/10.1186/s12883-020-02028-0.

Additional file 1. Summary statistics of neurological conditions

considered.

\section{Abbreviations}

AUROC: Area under the receiver operator characteristic curve; NNE: Number needed to evaluate; CPT: Current Procedural Terminology; ICD-10CM: International Classification of Diseases, Tenth Revision, Clinical Modification

\section{Acknowledgements}

Not Applicable.

\section{Authors' contributions}

$\mathrm{RO}$ assisted with model interpretation and selection. Wrote initial drafts of the paper, reviewed and revised final draft. WF assisted in model interpretation, wrote initial drafts of the paper, reviewed and revised the final draft. WW wrote initial drafts of the paper, reviewed and revised the final draft. She assisted with submission of the manuscript and other related tasks. ST conceived of the study, assisted in model interpretation, reviewed and revised final draft of the paper. LE led data analysis and model development, wrote initial drafts of the paper, reviewed and revised final draft. All authors have read and approved the manuscript.

Funding

None.

Availability of data and materials

Database used for this study is closed to public access.

Ethics approval and consent to participate

The need for patient consent was waived by Children's Hospital of Orange County Institutional Review Board (IRB) and patient data were de-identified prior to data collection. This study was approved by the Children's Hospital of Orange County IRB (IRB Number 180857).

Consent for publication

Not applicable.

Competing interests

None of the authors have conflict of interest to disclose.

Author details

${ }^{1}$ Sharon Disney Lund Medical Intelligence and Innovation Institute (MI3) at CHOC, Orange, California, USA. ${ }^{2}$ Children's Hospital of Orange County (CHOC), Orange, California, USA. ${ }^{3}$ Department of Emergency Medicine, University of California - Irvine, Irvine, California, USA. ${ }^{4}$ Neuroscience, Hamilton College, Clinton, New York, USA. ${ }^{5}$ Department of Pediatrics, University of California - Irvine, Irvine, California, USA. ${ }^{6}$ Chapman University School of Computational and Data Science, Orange, California, USA. 
Received: 25 July 2020 Accepted: 14 December 2020

Published online: 05 January 2021

\section{References}

1. Cohen E, Berry JG, Camacho X, Anderson G, Wodchis W, Guttmann A. Patterns and costs of health care use of children with medical complexity. Pediatrics. 2012;130(6):e1463-70.

2. Leary JC, Price LL, Scott CER, Kent D, Wong JB, Freund KM. Developing prediction models for 30-day unplanned readmission among children with medical complexity. Hosp Pediatr. 2019;9(3):201-8.

3. Hong A, Shah Y, Singh K, Karkare S, Kothare S. Characteristics and predictors of 7- and 30-day hospital readmissions to pediatric neurology. Neurology. 2019;92(16):e1926 LP-e1932. https://doi.org/10.1212/WNL. 0000000000007280.

4. Berry JG, Toomey SL, Zaslavsky AM, et al. Pediatric readmission prevalence and variability across hospitals. JAMA. 2013;309(4):372-80.

5. Simon TD, Berry J, Feudtner $C$, et al. Children with complex chronic conditions in inpatient hospital settings in the United States. Pediatrics. 2010;126(4):647-55.

6. Kocher RP, Adashi EY. Hospital readmissions and the affordable care act: paying for coordinated quality care. JAMA. 2011;306(16):1794-5. https://doi. org/10.1001/jama.2011.1561.

7. Williams MV. A requirement to reduce readmissions: take Care of the Patient, not just the DiseaseA program to reduce hospital readmissions. JAMA. 2013;309(4):394-6. https://doi.org/10.1001/jama.2012.233964.

8. Guterman EL, Douglas VC, Shah MP, Parsons T, Barba J, Josephson SA. National characteristics and predictors of neurologic 30-day readmissions. Neurology. 2016;86(7):669 LP-675. https://doi.org/10.1212/WNL. 0000000000002379.

9. Ehwerhemuepha L, Pugh K, Grant A, et al. A statistical-learning model for unplanned 7-day readmission in pediatrics. Hosp Pediatr. 2020;10(1):43-51.

10. Ehwerhemuepha L, Finn S, Rothman MJ, Rakovski C, Feaster W. A novel model for enhanced prediction and understanding of unplanned 30-day pediatric readmission. Hosp Pediatr. 2018;8(9):578-87.

11. Heinze $\mathrm{G}$, Schemper M. A solution to the problem of separation in logistic regression. Stat Med. 2002;21(16):2409-19.

12. O'brien RM. A caution regarding rules of thumb for variance inflation factors. Qual Quant. 2007;41(5):673-90.

13. Bozdogan H. Model selection and Akaike's information criterion (AIC): The general theory and its analytical extensions. Psychometrika. 1987;52(3):34570.

14. Ehwerhemuepha L, Gasperino G, Bischoff N, Taraman S, Chang A, Feaster W. HealtheDataLab - a cloud computing solution for data science and advanced analytics in healthcare with application to predicting multi-center pediatric readmissions. BMC Med Inform Decis Mak. 2020;20(1):1-12. https:// doi.org/10.1186/s12911-020-01153-7.

15. R Core Team. R: A Language and Environment for Statistical Computing 2017. https://www.r-project.org/.

16. Kansagara D, Englander $H$, Salanitro A, et al. Risk prediction models for hospital readmission: a systematic review. JAMA. 2011;306(15):1688-98.

\section{Publisher's Note}

Springer Nature remains neutral with regard to jurisdictional claims in published maps and institutional affiliations.

Ready to submit your research? Choose BMC and benefit from:

- fast, convenient online submission

- thorough peer review by experienced researchers in your field

- rapid publication on acceptance

- support for research data, including large and complex data types

- gold Open Access which fosters wider collaboration and increased citations

- maximum visibility for your research: over $100 \mathrm{M}$ website views per year

At $\mathrm{BMC}$, research is always in progress.

Learn more biomedcentral.com/submissions 